J. Chabrowski

Nagoya Math. J.

Vol. 93 (1984), 109-131

\title{
ON NON-LOCAL PROBLEMS FOR PARABOLIC EQUATIONS
}

\section{J. CHABROWSKI}

The main purposes of this paper are to investigate the existence and the uniqueness of a non-local problem for a linear parabolic equation

$$
L u=\sum_{i, j=1}^{n} a_{i j}(x, t) \frac{\partial^{2} u}{\partial x_{i} \partial x_{j}}+\sum_{i=1}^{n} b_{i}(x, t) \frac{\partial u}{\partial x_{i}}+c(x, t) u-\frac{\partial u}{\partial t}=f(x, t)
$$

in a cylinder $D=\Omega \times(0, T]$. Given functions $\beta_{i}(i=1, \cdots, N)$ on $\Omega$ and numbers $T_{i} \in(0, T](i=1, \cdots, N)$, the problem in question is to find a solution $u$ of (1) satisfying the following conditions

$$
\begin{gathered}
u(x, t)=\phi(x, t) \quad \text { on } \Gamma, \\
u(x, 0)+\sum_{i=1}^{N} \beta_{i}(x) u\left(x, T_{i}\right)=\Psi(x) \quad \text { on } \Omega,
\end{gathered}
$$

where $f, \phi$ and $\Psi$ are given functions and $\Gamma$ denotes the lateral surface of $D$, i.e., $\Gamma=\partial \Omega \times[0, T]$.

In Section 1 we establish the maximum principle associated with the problem described by (1), (2) and (3). Theorem 1 leads immediately to the uniqueness of solution of the problem (1), (2) and (3) as well as to an estimate of the solution in terms of $f, \phi$ and $\Psi$. We also briefly discuss certain properties of the solutions related to the behaviour of the coefficients $\beta_{i}(i=1, \cdots, N)$. In Theorem 5 of Section 2 we establish the existence of the solution in a bounded cylinder. The results are then applied to derive the existence and the uniqueness of solution of the nonlocal problem in an unbounded cylinder (Section 3). In Section 4 we establish an integral representation of solutions and give a construction of the solution of a non-local problem in $R_{n} \times(0, T]$ with $\Psi \in L^{2}\left(R_{n}\right)$. In the last section we modify the condition (3) by replacing a finite sum by an infinite series and briefly discuss the uniqueness and the existence of solution of the resulting problem. Theorems of Sections 1 and 2 of this

Received September 17, 1982. 
paper extend and improve earlier results obtained by Kerefov [3] and Vabishchevich [6], where historical references can be found. They only considered the case $N=1$.

1. Let $D=\Omega \times(0, T]$, where $\Omega$ is a bounded domain in $R_{n}$. By $\Gamma$ we denoted the lateral surface of $D$, i.e., $\Gamma=\partial \Omega \times[0, T]$.

Throughout this section we make the following assumption

(A) The coefficients $a_{i j}, b_{i}$ and $c$ are continuous on $D$ and moreover

$$
\sum_{i, j=1}^{n} a_{i j}(x, t) \xi_{i} \xi_{j}>0
$$

for all vectors $\xi \neq 0$ and $(x, t) \in D$.

By $C^{2,1}(D)$ we denote the set of functions $u$ continuous on $D$ with their derivatives $\partial u / \partial x_{i}, \partial^{2} u / \partial x_{i} \partial x_{i}(i, j=1, \cdots, n)$ and $\partial u / \partial t$ (at $t=T$ the derivative $\partial u / \partial t$ is understood as the left-hand derivative).

Lemma 1. Let $u \in C^{2,1}(D) \cap C(\bar{D})$. Suppose that $c(x, t) \leq 0$ on $D$ and $-1 \leq \sum_{i=1}^{N} \beta_{i}(x) \leq 0$ on $\Omega$ and $\beta_{i}(x) \leq 0$ on $\Omega(i=1, \cdots, N)$. If $L u \leq 0$ in $D, u(x, t) \geq 0$ on $\Gamma$ and $u(x, 0)+\sum_{i=1}^{N} \beta_{i}(x) u\left(x, T_{i}\right) \geq 0$ on $\Omega$, then $u(x, t)$ $\geq 0$ on $\bar{D}$.

Proof. Assume that $u<0$ at some point of $\bar{D}$, then there exists a point $\left(x_{0}, t_{0}\right) \in \bar{D}$ such that $u\left(x_{0}, t_{0}\right)=\min _{\bar{D}} u(x, t)<0$. By the strong maximum principle $\left(x_{0}, t_{0}\right)=\left(x_{0}, 0\right)$ with $x_{0} \in \Omega$ (see Friedman [2] Chap. 2 or Protter and Weinberger [5] Chap. 3). Thus, we find that

$$
0 \leq u\left(x_{0}, 0\right)+\sum_{i=1}^{N} \beta_{i}\left(x_{0}\right) u\left(x_{0}, T_{i}\right) \leq u\left(x_{0}, 0\right)\left[1+\sum_{i=1}^{N} \beta_{i}\left(x_{0}\right)\right] \text {. }
$$

Hence $u\left(x_{0}, 0\right) \geq 0$ provided $1+\sum_{i=1}^{N} \beta_{i}\left(x_{0}\right)>0$ and we get a contradiction.

In the case $\sum_{i=1}^{N} \beta_{i}\left(x_{0}\right)=-1$ we put $u\left(x_{0}, T_{k}\right)=\min _{i=1, \cdots, N} u\left(x_{0}, T_{i}\right)$, then

$$
\begin{aligned}
u\left(x_{0}, 0\right)-u\left(x_{0}, T_{k}\right) & =u\left(x_{0}, 0\right)+u\left(x_{0}, T_{k}\right) \sum_{i=1}^{N} \beta_{i}\left(x_{0}\right) \\
& \geq u\left(x_{0}, 0\right)+\sum_{i=1}^{N} \beta_{i}(x) u\left(x_{0}, T_{i}\right) \geq 0 .
\end{aligned}
$$

Hence $u$ takes on a negative minimum at $\left(x_{0}, T_{k}\right) \in D$. This contradiction completes the proof.

Corollary. Suppose that the assumptions of Lemma 1 hold. If $L \geq$ 0 in $D, u(x, t) \leq 0$ an $\Gamma$ and $u(x, 0)+\sum_{i=1}^{N} \beta_{i}(x) u\left(x, T_{i}\right) \leq 0$ on $\Omega$, then $u(x, t) \leq 0$ on $\bar{D}$. 
Now we can state the main result of this section.

Theorem 1. Let $u \in C^{2,1}(D) \cap C(\bar{D})$ be a solution of the problem (1), (2) and (3) with $f$, $\phi$ and $\Psi$ continuous on $\bar{D}, \Gamma$ and $\bar{\Omega}$ respectively. Suppose that $c(x, t) \leq-c_{0}$ in $D$, where $c_{0}$ is a positive constant. Assume further that $-1 \leq \sum_{i=1}^{N} \beta_{i}(x) \leq 0$ and $\beta_{i}(x) \leq 0(i=1, \cdots, N)$ on $\Omega$. Then

$$
\begin{aligned}
|u(x, t)| \leq & \frac{2}{c_{0}} e^{\left(c_{0} / 2\right) T} \sup _{D}|f(x, t)|+e^{\left(c_{0} / 2\right) T} \sup _{\Gamma}|\phi(x, t)| \\
& +\left(1-e^{-\left(c_{0} / 2\right) T_{k}}\right)^{-1} \sup _{\Omega}|\Psi(x)|
\end{aligned}
$$

for all $(x, t) \in \bar{D}$, where $T_{k}=\min _{i=1, \ldots, N} T_{i}$.

Proof. We first suppose that $-1<-\beta_{0} \leq \sum_{i=1}^{N} \beta_{i}(x) \leq 0$ on $\Omega$, where $\beta_{0}$ is a positive constant. Let $M=\sup _{b}|f(x, t)|, M_{1}=\sup _{\Gamma}|\phi(x, t)|, M_{2}=$ $\sup _{\Omega}|\Psi(x)|$ and put

$$
v(x, t)=u(x, t)-\frac{M}{c_{0}}-M_{1}-\frac{M_{2}}{1-\beta_{0}} .
$$

Then

$$
L v=f-\frac{c}{c_{0}} M-c M_{1}-\frac{c M_{2}}{1-\beta_{0}} \geq c_{0} M_{1}+\frac{c_{0}}{1-\beta_{0}} M_{2}>0
$$

in $D, v(x, t) \leq 0$ on $T$ and

$$
\begin{gathered}
v(x, 0)+\sum_{i=1}^{N} \beta_{i}(x) v\left(x, T_{i}\right)=\Psi(x)-\frac{M}{c_{0}}-M_{1}-\frac{M_{2}}{1-\beta_{0}} \\
-\left(\frac{M}{c_{0}}+M_{1}+\frac{M_{2}}{1-\beta_{0}}\right) \sum_{i=1}^{N} \beta_{i}(x) \leq\left(\frac{M}{c_{0}}+M_{1}\right)\left(\beta_{0}-1\right) \\
+M_{2}\left(1-\frac{1}{1-\beta_{0}}+\frac{\beta_{0}}{1-\beta_{0}}\right)<0
\end{gathered}
$$

on $\Omega$. It follows from Lemma 1 that $v \leq 0$ on $D$. Similarly we can establish the inequality $u(x, t) \geq-\left(M / c_{0}\right)-M_{1}-M_{2} /\left(1-\beta_{0}\right)$ for $(x, t) \in \bar{D}$ considering the auxiliary function

$$
w(x, t)=u(x, t)+\frac{M}{c_{0}}+M_{1}+\frac{M_{2}}{1-\beta_{0}}
$$

In the general case we put $u(x, t)=e^{-\left(c_{0} / 2\right) t} z(x, t)$. Then $z$ satisfies the equation 


$$
\begin{array}{r}
\sum_{i, j=1}^{n} a_{i j}(x, t) \frac{\partial^{2} z}{\partial x_{i} \partial x_{j}}+\sum_{i=1}^{n} b_{i}(x, t) \frac{\partial z}{\partial x_{i}}+\left(c(x, t)+\frac{c_{0}}{2}\right) z-\frac{\partial z}{\partial t} \\
=e^{\left(c_{0} / 2\right) t} f(x, t)
\end{array}
$$

in $D$ with $c(x, t)+c_{0} / 2 \leq-\left(c_{0} / 2\right)$ in $D$,

$$
z(x, t)=e^{\left(c_{0} / 2\right) t} \phi(x, t) \quad \text { on } \Gamma
$$

and

$$
z(x, 0)+\sum_{i=1}^{N} \beta_{i}(x) e^{-\left(c_{0} / 2\right) T_{i}} z\left(x, T_{i}\right)=\Psi(x) \text { on } \Omega
$$

It is clear that $-e^{-\left(c_{0} / 2\right) T_{k}} \leq \sum_{i=1}^{N} \beta_{i}(x) e^{-\left(c_{0} / 2\right) T_{i}} \leq 0$ on $\Omega$ and the estimate easily follows.

Theorem 1 and a classical maximum principle for solutions of parabolic equations allow us to compare a solution of the problem (1), (2) and (3) with a solution of an initial boundary value problem.

TheOREm 2. Suppose that the assumptions of Theorem 1 hold. Let $u \in C^{2,1}(D) \cap C(\bar{D})$ be a solution of the problem (1), (2) and (3), and $v \in$ $C^{2,1}(D) \cap C(\bar{D})$ a solution of (1) satisfying the initial boundary value conditions $v(x, t)=\phi(x, t)$ on $\Gamma$ and $v(x, 0)=\Psi(x)$ on $\Omega$. Then

$$
\begin{aligned}
|u(x, t)-v(x, t)| \leq \sup _{\Omega} \sum_{i=1}^{N}\left|\beta_{i}(x)\right|\left[\frac{2}{c_{0}} e^{\left(c_{0} / 2\right) T} \sup _{D}|f(x, t)|\right. \\
\left.+e^{\left(c_{0} / 2\right) T} \sup _{\Gamma}|\phi(x, t)|+\left(1-e^{-\left(c_{0} / 2\right) T_{k}}\right)^{-1} \sup _{\Omega}|\Psi(x)|\right]
\end{aligned}
$$

for all $(x, t) \in \bar{D}$.

In particular if $\beta_{i}=\beta_{\nu}^{i}(x)(i=1, \cdots, N)$ where $\beta_{\nu}^{i} \rightarrow 0$ uniformly as $\nu \rightarrow \infty$ for all $i$, then the corresponding sequence $u_{\nu}$ of solutions of the problem (1), (2) and (3) converges uniformly to $v$ in $\bar{D}$.

Theorem 3. Let $c(x, t) \leq 0$ in $D$ and assume that $-1 \leq \sum_{i=1}^{N} \beta_{i}^{j}(x) \leq 0$ $(j=1,2)$ and that $\beta_{i}^{1}(x) \leq \beta_{i}^{2}(x) \leq 0(i=1, \cdots, N)$ on $\Omega$. Suppose further that $f \leq 0, \phi \geq 0$ and $\Psi \geq 0$ on $D, \Gamma$ and $\bar{\Omega}$ respectively. If $u_{1}$ and $u_{2}$ are solutions belonging to $C^{2,1}(D) \cap C(\bar{D})$ of the problem (1), (2) and (3) with $\beta_{i}=\beta_{i}^{1}(x)(i=1, \cdots, N)$ and $\beta_{i}=\beta_{i}^{2}(x)(i=1, \cdots, N)$ respectively, then $u_{1}(x, t) \geq u_{2}(x, t)$ on $\bar{D}$.

Proof. We put $w(x, t)=u_{1}(x, t)-u_{2}(x, t)$, then $L w=0$ in $D, w(x, t)$ $=0$ on $\Gamma$ and 


$$
w(x, 0)+\sum_{i=1}^{N} \beta_{i}^{1}(x) w\left(, x, T_{i}\right)=\sum_{i=1}^{N}\left(\beta_{i}^{2}(x)-\beta_{i}^{1}(x)\right) u_{2}\left(x, T_{i}\right) \text { on } \Omega .
$$

Since $u_{2}(x, t) \geq 0$ on $\bar{D}$, it follows from Lemma 1 , that $w(x, t) \geq 0$ for all $(x, t) \in \bar{D}$.

Lemma 1 yields the uniqueness of solutions of the problem (1), (2) and (3) under the assumptions that $\beta_{i}(x) \leq 0(i=1, \cdots, N)$ and $-1 \leq$ $\sum_{i=1}^{N} \beta_{i}(x) \leq 0$ on $\Omega$. Vabishchevich [6] pointed out, without giving any proof, that in the case $N=1$ the uniqueness can be proved under the assumption $|\beta(x)| \leq 1$ on $\Omega$. For the sake of completeness we include the proof of uniqueness under the assumption $\sum_{i=0}^{N}\left|\beta_{i}(x)\right| \leq 1$ on $\Omega$.

Theorem 4. Suppose that $c(x, t) \leq 0$ on $D$ and $\sum_{i=1}^{N}\left|\beta_{i}(x)\right| \leq 1$ on $\Omega$. Then the problem (1), (2) and (3) has at most one solution in $C^{2,1}(D) \cap C(\bar{D})$.

Proof. Let $u$ be a solution of the homogeneous problem

$$
\begin{aligned}
L u=0 & \text { in } D \\
u(x, t)=0 & \text { on } \Gamma
\end{aligned}
$$

and

$$
u(x, 0)+\sum_{i=1}^{N} \beta_{i}(x) u\left(x, T_{i}\right)=0 \quad \text { on } \Omega .
$$

Suppose that $u \neq \equiv 0$. We also many assume that there exists a point in $\left(x_{0}, t_{0}\right) \in \bar{D}$ such that $u\left(x_{0}, t_{0}\right)=\min _{\bar{D}} u(x, t)<0$. It is clear that $\left(x_{0}, t_{0}\right)=$ $\left(x_{0}, 0\right)$ with $x_{0} \in \Omega$. We can assume that $\left|u\left(x_{0}, T_{1}\right)\right|=\max _{i=1, \ldots, N}\left|u\left(x_{0}, T_{i}\right)\right|$ $>0$, since otherwise there is nothing to prove. Obviously,

$$
\left|u\left(x_{0}, 0\right)\right| \leq\left|u\left(x_{0}, T_{1}\right)\right| \sum_{i=1}^{N}\left|\beta_{i}\left(x_{0}\right)\right| \leq\left|u\left(x_{0}, T_{1}\right)\right| .
$$

If $u\left(x_{0}, T_{1}\right)<0$ then $u\left(x_{0}, T_{1}\right) \leq u\left(x_{0}, 0\right)$. Hence $u$ attains its negative minimum at $\left(x_{0}, T_{1}\right)$ and we get a contradiction, therefore $u\left(x_{0}, T_{1}\right)>0$. Thus there exists a point $\left(x_{1}, t_{1}\right) \in \bar{D}$ such that $u\left(x_{1}, t_{1}\right)=\max _{\bar{D}} u(x, t)>0$. Again $\left(x_{1}, t_{1}\right)=\left(x_{1}, 0\right)$ with $x_{1} \in \Omega$. Put $\left|u\left(x_{1}, T_{s}\right)\right|=\max _{i=1, \ldots, N}\left|u\left(x_{1}, T_{i}\right)\right|$. We may assume that $\left|u\left(x_{1}, T_{s}\right)\right|>0$, since otherwise there is nothing to prove. Now we must distinguish two cases

$$
\left|u\left(x_{0}, 0\right)\right|<u\left(x_{1}, 0\right) \quad \text { or } \quad u\left(x_{1}, 0\right) \leq\left|u\left(x_{0}, 0\right)\right| .
$$

In the first case we have

$$
\left|u\left(x_{0}, 0\right)\right|<u\left(x_{1}, 0\right) \leq\left|u\left(x_{1}, T_{s}\right)\right| \sum_{i=1}^{N}\left|\beta_{i}(x)\right| \leq\left|u\left(x_{1}, T_{s}\right)\right|,
$$


consequently if $u\left(x_{1}, T_{s}\right)<0$ then $u\left(x_{0}, 0\right)>u\left(x, T_{s}\right)$. Hence $u$ takes on a positive minimum at $\left(x_{1}, T_{s}\right) \in D$ and we get a contradiction. On the other hand if $u\left(x_{1}, T_{s}\right)>0$ we have $u\left(x_{1}, 0\right) \leq u\left(x_{1}, T_{s}\right)$. Hence $u$ attains a positive maximum at $\left(x_{1}, T_{s}\right)$ and we arrive at a contradiction. Similarly in the second case we obtain

$$
u\left(x_{1}, 0\right) \leq\left|u\left(x_{0}, 0\right)\right| \leq u\left(x_{0}, T_{1}\right) \sum_{i=1}^{N}\left|\beta_{i}\left(x_{0}\right)\right| \leq u\left(x_{0}, T_{1}\right)
$$

and $u$ takes on a positive maximum at $\left(x_{0}, T_{1}\right) \in D$. This contradiction completes the proof.

2. For the existence theorem we shall need the following assumptions

$\left(A_{1}\right)$ There exist positive constants $\lambda_{0}$ and $\lambda_{1}$ such that, for any vector $\xi \in R_{n}$

$$
\lambda_{0}|\xi|^{2} \leq \sum_{i, j=1}^{n} a_{i j}(x, t) \xi_{i} \xi_{j} \leq \lambda_{1}|\xi|^{2}
$$

for all $(x, t) \in D$.

$\left(\mathrm{A}_{2}\right)$ The coefficients $a_{i j}, b_{i}(i, j=1, \cdots, n), c$ and $f$ are Hölder continuous in $D$ (exponent $\alpha$ ).

$\left(\mathrm{A}_{3}\right)$ The functions $\phi, \Psi$ and $\beta_{i}(i=1, \cdots, N)$ are continuous respectively on $\Gamma, \bar{\Omega}$ and $\bar{Q}$ and, in addition,

$$
\Psi(x)=\phi(x, 0)+\sum_{i=1}^{N} \beta_{1}(x) \phi\left(x, T_{i}\right)
$$

for all $x \in \partial \Omega$.

Moreover we assume that $\partial \Omega \in C^{2+\alpha}$.

Theorem 5. Let $c(x, t) \leq-c_{0}$, where $c_{0}$ is a positive constant and assume that $-1 \leq \sum_{i=1}^{N} \beta_{i}(x) \leq 0$ and $\beta_{i}(x) \leq 0(i=1, \cdots, N)$ on $\bar{\Omega}$. Then there exists a unique solution in $C^{2,1}(D) \cap C(\bar{D})$ of the problem (1), (2) and (3).

Proof. We first assume that $\phi \equiv 0$ on $\Gamma$, then by the condition $\left(\mathrm{A}_{3}\right)$ $\Psi(x)=0$ on $\partial \Omega$. We try to find a solution in the form

$$
u(x, t)=\int_{\Omega} G(x, t ; y, 0) u(y, 0) d y-\int_{0}^{t} \int_{\Omega} G(x, t ; y, \tau) f(y, \tau) d y d \tau,
$$

where $u(y, 0)$ is to be determined and $G$ denotes the Green function for the operator $L$. The condition (3) leads to the Fredholm integral equa- 
tion of the second kind

$$
\begin{aligned}
u(x, 0) & +\sum_{i=1}^{N} \beta_{i}(x) \int_{\Omega} G\left(x, T_{i} ; y, 0\right) u(y, 0) d y \\
& =\Psi(x)+\sum_{i=1}^{N} \beta_{i}(x) \int_{0}^{T_{i}} G\left(x, T_{i} ; y, \tau\right) f(y, \tau) d y d \tau .
\end{aligned}
$$

Applying Theorem 4 it is easy to show that the corresponding homogeneous equation only has a trivial solution in $L^{2}(\Omega)$. Hence there exists a unique solution $u(\cdot, 0)$ in $L^{2}(\Omega)$ of the equation (7). Since $\Psi(x)=0$ on $\partial \Omega$, it follows from the properties of the Green function that $u(\cdot, 0) \in C(\bar{\Omega})$ and $u(x, 0)=0$ on $\partial \Omega$. Consequently the formula (6) gives a solution in this case.

Suppose next $\phi \neq \equiv$, but assume that there exists a function $\Phi \in \bar{C}^{2+\alpha}(D)$ such that $\Phi=\phi$ on $\Gamma$. Introducing $v=u-\Phi$ we then immediately obtain, by the previous result, the existence of a solution $v$ to $L v=f-L \Phi$ which vanishes on $\Gamma$ and satisfies the condition

$$
v(x, 0)+\sum_{i=1}^{N} \beta_{i}(x) v\left(x, T_{i}\right)=\Psi(x)-\Phi(x, 0)-\sum_{i=1}^{N} \beta_{i}(x) \Phi\left(x, T_{i}\right)
$$

for all $x \in \Omega$. Then assertions for $u$ then follow.

We finally consider the general case, where $\phi$ is only assumed to be continuous. By Theorem 2 in Friedman [2] (p. 60) and the Weierstrass approximation theorem there exists a sequenece of polynomials $\Phi_{m}$ on $\bar{D}$ which approximates $\phi$ uniformly on $\Gamma$. Now we define a function $\Psi_{m}$ on $\partial \Omega$ by the following formula

$$
\Psi_{m}(x)=\Phi_{m}(x, 0)+\sum_{i=1}^{N} \beta_{i}(x) \Phi_{m}\left(x, T_{i}\right)
$$

for $x \in \partial \Omega$. Since $\lim _{m \rightarrow \infty} \Psi_{m}=\Psi$ uniformly on $\partial \Omega$, one can construct a sequence of functions $\left\{\tilde{\Psi}_{m}\right\}$ in $C(\bar{\Omega})$ such that $\lim _{m \rightarrow \infty} \tilde{\Psi}_{m}=\Psi$ uniformly on $\bar{\Omega}$ and $\tilde{\Psi}_{m}=\Psi_{m}$ on $\partial \Omega$ for all $m$. By what we have already proved there exist solutions to the problem

$$
\begin{aligned}
L u_{m} & =f \text { in } D, \\
u_{m}(x, t) & =\Phi_{m}(x, t) \text { on } \Gamma,
\end{aligned}
$$

and

$$
u_{m}(x, 0)+\sum_{i=1}^{N} \beta_{i}(x) u_{m}\left(x, T_{i}\right)=\tilde{\Psi}_{m}(x) \quad \text { on } \Omega
$$


By Theorem 1 (the inequality (4)) the sequence $u_{m}(x, t)$ is uniformly convergent on $\bar{D}$ to a function $u$. It is clear that $u$ satisfies the conditions (2) and (3). Using Friedman-Schauder interior estimates (Friedman [2], Theorem 5 p. 64) one can easily prove that $u$ satisfies the equation (1).

Remark. In the above proof we followed the argument used in the proof of Theorem 9 in Friedman [2] (p. 70-71). For the definition of the space $\bar{C}^{2+\alpha}(D)$ see Friedman [2] (p. 61-62).

Theorem 6. Suppose that $\sum_{i=1}^{N}\left|\beta_{i}(x)\right| \leq 1$ on $\Omega, c(x, t) \leq 0$ on $D$ and $\phi \equiv 0$ on $\Gamma$. Then the problem (1), (2) and (3) has a unique solution in $C^{2,1}(D) \cap C(\bar{D})$.

Proof. A solution to this problem is given by the formula

$$
u(x, t)=\int_{\Omega} G(x, t ; y, 0) u(y, 0) d y-\int_{0}^{t} \int_{\Omega} G(x, t ; y, \tau) f(y, \tau) d y d \tau,
$$

where $u(x, 0)$ is a solution of the Fredholm integral equation of the second kind

$$
\begin{aligned}
& u(x, 0)+\sum_{i=1}^{N} \beta_{i}(x) \int_{\Omega} G\left(x, T_{i} ; y, 0\right) u(y, 0) d y \\
& =\Psi(x)+\sum_{i=1}^{N} \beta_{i}(x) \int_{0}^{T_{i}} \int_{\Omega} G\left(x, T_{i} ; y, \tau\right) f(y, \tau) d y d \tau .
\end{aligned}
$$

3. In this section we investigate the existence of a solution of the problem (1), (2) and (3) in an unbounded cylinder. Let $D=\Omega \times(0, T]$, where $\Omega$ is an unbounded domain in $R_{n}$.

In the next theorem we give a general method of constructing a solution. We shall need the following assumptions

$\left(\mathrm{B}_{1}\right)$ The coefficients $a_{i j}, b_{i}(i, j=1, \cdots, n)$ and $c$ are continuous on $D$ and moreover

$$
\sum_{i, j=1}^{n} a_{i j}(x, t) \xi_{i} \xi_{j}>0
$$

for every $(x, t) \in D$ and any vector $\xi \neq 0, a_{i j}=a_{j i}(i, j=1, \cdots, n)$.

$\left(\mathrm{B}_{2}\right)$ There exists a family of positive function $H(x, \delta)\left(0<\delta \leq \delta_{0}\right)$ defined on $\Omega$ with properties:

(i ) $H \in C^{2}(\Omega) \cap C(\bar{\Omega})$ for $0<\delta \leq \delta_{0}$ and $L H \leq-c_{0} H$ for all $(x, t) \in D$ and $0<\delta \leq \delta_{0}$, where $c_{0}$ is a positive constant,

(ii) $\lim _{|x| \rightarrow \infty} \frac{H\left(x, \delta_{1}\right)}{H\left(x, \delta_{2}\right)}=0$ for $0<\delta_{1}<\delta_{2} \leq \delta_{0}$, 
(iii) there exists a positive constant $\kappa$ such that

$$
H\left(x, \delta_{1}\right) \leq \kappa H\left(x, \delta_{2}\right)
$$

for all $x \in \Omega$ and $0<\delta_{1}<\delta_{2} \leq \delta_{0}$.

For a sequence $\left\{R_{p}\right\}$ of positive numbers we define

$$
\Omega_{p}=\Omega \cap\left\{x:|x|<R_{p}\right\}, \quad \Gamma_{p}=\partial \Omega_{p} \times[0, T] \quad \text { and } \quad D_{p}=\Omega_{p} \times(0, T] .
$$

$\left(\mathrm{B}_{3}\right)$ There exists a sequence of positive numbers $R_{p}$ converging to $\infty$ as $p \rightarrow \infty$ such that the problem (1), (2) and (3) is solvable on every $D_{p}$, i.e. for every bounded and Hölder continuous function $f$ on $D_{p}$ and all continuous functions $\phi$ and $\Psi$ on $\Gamma_{p}$ and $\bar{\Omega}_{p}$ respectively, and satisfying the condition

$$
\Psi(x)=\phi(x, 0)+\sum_{i=1}^{N} \beta_{i}(x) \phi\left(x, T_{i}\right) \quad \text { on } \partial \Omega_{p}
$$

the problem

$$
\begin{aligned}
L u & =f \text { in } D_{p}, \\
u(x, t) & =\phi(x, t) \quad \text { on } \Gamma_{p}
\end{aligned}
$$

and

$$
u(x, 0)+\sum_{i=1}^{N} \beta_{i}(x) u\left(x, T_{i}\right)=\Psi(x) \text { on } \Omega_{p}
$$

has a unique solution in $C^{2,1}\left(D_{p}\right) \cap C\left(\bar{D}_{p}\right)$.

We shall say that a function $u$ defined on $D$ belongs to $E_{H}(D)$ if there exist positive constants $M$ and $\delta<\delta_{0}$ such that $|u(x, t)| \leq M H(x, \delta)$ for all $(x, t) \in D$.

We shall say that a function $v$ defined on $\Omega$ belongs to $E_{H}(\Omega)$ if there exist positive constants $M$ and $\delta<\delta_{0}$ such that $|v(x)| \leq M H(x, \delta)$ for all $x \in \Omega$.

We are now in a position to construct a solution of the problem (1), (2) and (3). The construction given in the proof of Theorem 7 below is a modification of the method used by Krzyżański [4] to solve the Cauchy problem for parabolic equations.

Theorem 7. Suppose that the assumptions $\left(\mathrm{B}_{1}\right),\left(\mathrm{B}_{2}\right)$ and $\left(\mathrm{B}_{3}\right)$ hold. Let $-1 \leq \sum_{i=1}^{N} \beta_{i}(x) \leq 0$ and $\beta_{i}(x) \leq 0(i=1, \cdots, N)$ on $\Omega$. Assume that $f \in E_{H}(D)$ is an Hölder continuous function, that $\phi \in E_{H}(D)$ and $\Psi \in E_{H}(\Omega)$ are continuous functions on $\bar{D}$ and $\bar{\Omega}$ respectively and moreover that 


$$
\Psi(x)=\phi(x, 0)+\sum_{i=1}^{N} \beta_{i}(x) \phi\left(x, T_{i}\right) \quad \text { on } \partial \Omega_{p}
$$

$p=1,2, \cdots$. Then the problem (1), (2) and (3) has a unique solution in $C^{2,1}(D) \cap C(\bar{D}) \cap E_{H}(D)$,

Proof. It is clear that there exist positive constants $M$ and $\delta \leq \delta_{0}$ such that

$$
\begin{gathered}
|\phi(x, t)| \leq M H(x, \delta), \quad|f(x, t)| \leq M H(x, \delta) \text { on } D, \\
|\Psi(x)| \leq M H(x, \delta) \text { on } \Omega .
\end{gathered}
$$

By the assumption $\left(\mathrm{B}_{3}\right)$ for every $p$ there exists a unique solution $u_{p}$ in $C^{2,1}(D) \cap C(\bar{D})$ of the problem

$$
\begin{aligned}
L u_{p} & =f \text { on } D_{p}, \\
u_{p}(x, t) & =\phi(x, t) \text { on } \Gamma_{p},
\end{aligned}
$$

and

$$
u_{p}(x, 0)+\sum_{i=1}^{N} \beta_{i}(x) u_{p}\left(x, T_{i}\right)=\Psi(x) \text { on } \bar{Q}_{p}
$$

Put

$$
u_{p}(x, t)=v_{p}(x, t) H(x, \delta) \quad p=1,2, \cdots
$$

for $(x, t) \in D_{p}$. Then for every $p\left|v_{p}(x, t)\right| \leq M$ on $\Gamma_{p}$,

$$
\left|v_{p}(x, 0)+\sum_{i=1}^{N} \beta_{i}(x) v_{p}\left(x, T_{i}\right)\right| \leq \frac{|\Psi(x)|}{H(x, \delta)} \leq M \quad \text { on } \Omega_{p}
$$

and

$$
\begin{aligned}
& \sum_{i, j=1}^{n} a_{i j}(x, t) \frac{\partial^{2} v_{p}}{\partial x_{i} \partial x_{j}}+\sum_{i=1}^{n}\left(b_{i}(x, t)+\right.\left.\frac{2}{H(x, \delta)} \sum_{j=1}^{n} a_{i j}(x, t) \frac{\partial H}{\partial x_{i}}\right) \frac{\partial v_{p}}{\partial x_{i}} \\
&+\frac{L H}{H} v_{p}-\frac{\partial v_{p}}{\partial t}=\frac{f(x, t)}{H(x, \delta)}
\end{aligned}
$$

in $D_{p}$. It follows from the assumption $\left(\mathrm{B}_{2} \mathrm{i}\right)$ and Theorem 1 that

$$
\left|v_{p}(x, t)\right| \leq\left[\frac{2}{c_{0}} e^{\left(c_{0} / 2\right) T}+e^{\left(c_{0} / 2\right) T}+\left(1-e^{-\left(c_{0} / 2\right) T_{k}}\right)^{-1}\right] M=M_{1}
$$

for all $(x, t) \in D_{p}, p=1,2, \cdots$, where $T_{k}=\min _{i} T_{i}$. Let $\delta<\delta_{1}<\delta_{0}$ and put

$$
u_{p}(x, t)=\bar{v}_{p}(x, t) H\left(x, \delta_{1}\right) \quad p=1,2, \cdots
$$


and

$$
u_{p q}(x, t)=u_{p}(x, t)-u_{q}(x, t)=H\left(x, \delta_{1}\right)\left[\bar{v}_{p}(x, t)-\bar{v}_{q}(x, t)\right]=H\left(x, \delta_{1}\right) \bar{v}_{p q}(x, t)
$$

for $p<q$. The function $\bar{v}_{p q}$ satisfies the homogeneous equation of the form (7) with $H(x, \delta)$ replaced by $H\left(x, \delta_{1}\right)$ and

$$
\bar{v}_{p q}(x, 0)+\sum_{i=1}^{N} \beta_{i}(x) \bar{v}_{p q}\left(x, T_{i}\right)=0
$$

on $\Omega_{p}$. Moreover

$$
\bar{v}_{p q}(x, t)=0 \quad \text { on }\left(\partial \Omega_{p} \cap \partial \Omega\right) \times(0, T]
$$

and

$$
\bar{v}_{p q}(x, t)=\frac{\phi_{p}(x, t)}{H\left(x, \delta_{1}\right)}-\frac{u_{q}(x, t)}{H\left(x, \delta_{1}\right)} \text { on } \Gamma_{p} \cap D
$$

consequently

$$
\left|\bar{v}_{p q}(x, t)\right| \leq\left(M+M_{1}\right) \sup _{\partial \Omega_{p}-\partial \Omega} \frac{H(x, \delta)}{H\left(x, \delta_{1}\right)} \quad \text { on } \Gamma_{p} .
$$

Let

$$
\varepsilon_{p}=\left(M+M_{1}\right) \sup _{\partial \Omega_{p} \partial \Omega} \frac{H(x, \delta)}{H\left(x, \delta_{1}\right)} .
$$

Thus by Theorem 1 we have

$$
\left|\bar{v}_{p q}(x, t)\right| \leq \varepsilon_{p} e^{\left(c_{0} / 2\right) T}
$$

on $\bar{D}_{p}$. By the assumption ( $\mathrm{B}_{2}$ ii) $\lim _{p \rightarrow \infty} \varepsilon_{p}=0$, hence $\bar{v}_{p}$ converges uniformly on every $\bar{D}_{s}$ to a function $\bar{v}$. Put $u(x, t)=\bar{v}(x, t) H\left(x, \delta_{1}\right)$ for $(x, t) \in \bar{D}$. Clearly $u \in E_{H}(D)$ is continuous on $\bar{D}$ and satisfies (2) and (3). To show that $u$ satisfies (1), fix an arbitrary index $p$ and consider the problem

$$
\begin{gathered}
L z=f \text { in } D_{p} \\
z(x, t)=u(x, t) \quad \text { on } \Gamma_{p}, \\
z(x, 0)+\sum_{i=1}^{N} \beta_{i}(x) z\left(x, T_{i}\right)=\Psi(x) \quad \text { on } \Omega_{p} .
\end{gathered}
$$

Since $u$ satisfies the condition (3), it is clear that

$$
u(x, 0)+\sum_{i=1}^{N} \beta_{i}(x) u\left(x, T_{i}\right)=\Psi(x) \quad \text { on } \partial \Omega_{p} .
$$

By the assumption $\left(\mathrm{B}_{3}\right)$ this problem has a unique solution $z$. Since $u_{q} \rightarrow u$ 
as $q \rightarrow \infty$ uniformly on $\bar{D}_{p}$, given $\varepsilon>0$ we can find $q_{0}$ such that $\mid u_{q}(x, t)$ $-u(x, t) \mid<\varepsilon$ for all $(x, t) \in \Gamma_{p}$ and $q \geq q_{0}$. Put

$$
u_{q}(x, t)-z(x, t)=w_{q}(x, t) H(x, \delta)
$$

for $(x, t) \in \bar{D}_{p}, q \geq q_{0}$. Then $w_{q}$ satisfies the homogeneous equation (8) in $D_{p}$ and the following conditions

$$
\left|w_{q}(x, t)\right| \leq \varepsilon \sup _{\Gamma_{p}} H(x, \delta)^{-1} \quad \text { on } \Gamma_{p}
$$

and

$$
w_{q}(x, 0)+\sum_{i=1}^{N} \beta_{i}(x) w_{q}\left(x, T_{i}\right)=0 \quad \text { on } \Omega_{p}
$$

By Theorem 1

$$
\left|w_{q}(x, t)\right| \leq \varepsilon e^{\left(c_{0} / 2\right) T} \sup _{\Gamma_{p}} H(x, \delta)^{-1}
$$

for all $(x, t) \in \bar{D}_{p}$. Letting $\varepsilon \rightarrow 0$ we obtain $u \equiv z$ on $D_{p}$ and the result follows. To establish uniqueness, let $u \in C^{2,1}(D) \cap C(\bar{D}) \cap E_{H}(D)$ be a solution of the problem (1), (2) and (3) with $f \equiv 0, \phi \equiv 0$ and $\Psi \equiv 0$. There exist positive constants $M$ and $\delta<\delta_{0}$ such that $|u(x, t)| \leq M H(x, \delta)$ in $D$. Choose $\delta<\delta_{1}<\delta_{0}$ and put

$$
u(x, t)=v(x, t) H\left(x, \delta_{1}\right) \text { on } D .
$$

By (ii) (the assumption $\left(\mathrm{B}_{2}\right)$ ) given $\varepsilon>0$ we can find a positive number $R$ such that

$$
|v(x, t)| \leq \varepsilon \quad \text { for }(x, t) \in \Omega \cap(|x| \geq R) \times(0, T] .
$$

By Theorem 1

$$
|v(x, t)| \leq \varepsilon e^{\left(c_{0} / 2\right) T}
$$

for all $(x, t) \in \bar{\Omega} \cap(|x| \leq R) \times[0, T]$ and the uniqueness easily follows.

To apply Theorem 7 we introduce the following assumptions

$\left(\mathrm{C}_{1}\right)$ The coefficients $a_{i j}, b_{i}(i, j=1, \cdots, n)$ and $c$ are bounded on $R_{n} \times[0, T]$ and Hölder continuous (with exponent $\alpha$ ) on every compact subset in $R_{n} \times[0, T]$ and moreover

$$
c(x, t) \leq-c_{0} \text { for all }(x, t) \in R_{n} \times[0, T],
$$

where $c_{0}$ is a positive constant.

$\left(\mathrm{C}_{2}\right)$ There exists positive constants $\lambda_{0}$ and $\lambda_{1}$ such that for any vector $\xi \in R_{n}$ 


$$
\lambda_{0}|\xi|^{2} \leq \sum_{i, j=1}^{n} a_{i j}(x, t) \xi_{i} \xi_{j} \leq \lambda_{1}|\xi|^{2}
$$

for all $(x, t) \in R_{n} \times(0, T], a_{i j}=a_{j i}(i, j=1, \cdots, n)$.

As an application of Theorem 7 we shall prove the existence of a solution $u$ of the equation (1) in $R_{n} \times(0, T]$ satisfying the condition

$$
u(x, 0)+\sum_{i=1}^{N} \beta_{i}(x) u\left(x, T_{i}\right)=\Psi(x) \text { on } R_{n} .
$$

It is clear that the function $H(x, \delta)=\prod_{i=1}^{n} \cosh \delta x_{i}$ has properties (i), (ii) and (iii) of the assumption $\left(\mathrm{B}_{2}\right)$ (with $\Omega=R_{n}$ ) provided $0<\delta<\delta_{0}$, where $\delta_{0}$ is sufficiently small.

In this situation

$E_{H}\left(R_{n} \times(0, T]\right)=\left\{u ; u\right.$ defined on $R_{n} \times(0, T]$ and $|u(x, t)| \leq M e^{\hat{\imath}|x|}$

for all $(x, t) \in R_{n} \times(0, T]$ and certain $M>0$ and $\left.0<\delta<\delta_{0}\right\}$,

similarly

$$
\begin{aligned}
& E_{H}\left(R_{n}\right)=\left\{v ; v \text { defined on } R_{n} \text { and }|v(x)| \leq M e^{\delta|x|}\right. \\
& \text { for all } \left.x \in R_{n} \text { and certain } M>0 \text { and } 0<\delta<\delta_{0}\right\} .
\end{aligned}
$$

Theorem 8. Suppose that the assumptions $\left(\mathrm{C}_{1}\right)$ and $\left(\mathrm{C}_{2}\right)$ holds. Let $\beta_{i} \in C\left(R_{n}\right), \beta_{i}(x) \leq 0(i=1, \cdots, N)$ and $-1 \leq \sum_{i=1}^{N} \beta_{i}(x) \leq 0$ on $R_{n}$. If $f \in$ $E_{H}\left(R_{n} \times(0, T]\right)$ is a Hölder continuous function on every compact subset of $R_{n} \times[0, T]$ and $\Psi \in E_{H}\left(R_{n}\right) \cap C\left(R_{n}\right)$, then the problem (1), (9) has a unique solution in $E_{H}\left(R_{n} \times(0, T]\right) \cap C^{2,1}\left(R_{n} \times(0, T] \cap C\left(R_{n} \times[0, T]\right)\right.$.

Proof. Let $\phi$ be a continuous function belonging to $E_{H}\left(R_{n} \times(0, T]\right)$ such that $\phi(x, 0)=\Psi(x)$ on $R_{n}$ and $\phi(x, t)=0$ on $R_{n} \times\left[T_{0}, T\right]$, where $T_{0}=\min _{i=1, \ldots, N} T_{i}$. By Theorem 5 the problem (1), (2) and (3) has a unique solution on every $D_{p}$. Applying Theorem 7 the result easily follows.

In the sequel we shall need the following result.

LEMMA 2. Suppose that the assumptions $\left(\mathrm{C}_{1}\right)$ and $\left(\mathrm{C}_{2}\right)$ hold in $R_{n} \times$ $(0, T]$. Let $\beta_{i} \in C\left(R_{n}\right)(i=1, \cdots, N),-1 \leq \sum_{i=1}^{N} \beta_{i}(x) \leq 0$ and $\beta_{i}(x) \leq 0(i=$ $1, \cdots, N)$ on $R_{n}$. Then for any bounded function $f$ on $R_{n} \times[0, T]$ and Hölder continuous on every compact subset of $R_{n} \times[0, T]$ and for any continuous and bounded function $\Psi$ on $R_{n}$ there exists a unique solution $u$ of the problem (1), (9) in $E_{H}\left(R_{n} \times(0, T]\right) \cap C^{2,1}\left(R_{n} \times(0, T]\right) \cap C\left(R_{n} \times[0, T]\right)$ such that 


$$
|u(x, t)| \leq \frac{2}{c_{0}} e^{\left(c_{0} / 2\right) T} \sup _{R_{n} \times[0, T]}|f(x, t)|+\left(1-e^{-\left(c_{0} / 2\right) T_{k}}\right)^{-1} \sup _{R_{n}}|\Psi(x)|
$$

for all $(x, t) \in R_{n} \times[0, T]$, where $T_{k}=\min _{i} T_{i}$.

Proof. We start with the following observation, the proof of which is routine,

$$
\text { if } u \in C^{2,1}\left(R_{n} \times(0, T]\right) \cap C\left(R_{n} \times[0, T]\right) \cap E_{H}\left(R_{n} \times(0, T]\right)
$$

and

$$
\begin{gathered}
L u \leq 0 \quad \text { in } R_{n} \times(0, T], \\
u(x, 0)+\sum_{i=1}^{N} \beta_{i}(x) u\left(x, T_{i}\right) \geq 0 \quad \text { on } R_{n}
\end{gathered}
$$

then $u \geq 0$ on $R_{n} \times[0, T]$.

We first suppose that $-1<-\beta_{0} \leq \sum_{i=1}^{N} \beta_{i}(x) \leq 0$ on $R_{n}$, where $\beta_{0}$ is a positive constant. Put

$$
v(x, t)=u(x, t)-\frac{M}{c_{0}}-\frac{M_{1}}{1-\beta_{0}},
$$

where

$$
M=\sup _{R_{n} \times[0, T]}|f(x, t)| \text { and } M_{1}=\sup _{R_{n}}|\Psi(x)| \text {. }
$$

Then

$$
L v=f-\frac{c}{c_{0}} M-\frac{c M_{1}}{1-\beta_{0}} \geq \frac{c_{0} M_{1}}{1-\beta_{0}}>0
$$

in $R_{n} \times(0, T]$ and

$$
\begin{aligned}
v(x, 0)+\sum_{i=1}^{N} \beta_{i}(x) v\left(x, T_{i}\right) & =\Psi(x)-\frac{M}{c_{0}}-\frac{M_{1}}{1-\beta_{0}}-\left(\frac{M}{c_{0}}+\frac{M_{1}}{1-\beta_{0}}\right) \sum_{i=1}^{N} \beta_{i}(x) \\
& \leq \frac{M}{c_{0}}\left(\beta_{0}-1\right)+M_{1}\left(1-\frac{1}{1-\beta_{0}}+\frac{\beta_{0}}{1-\beta_{0}}\right)<0
\end{aligned}
$$

on $R_{n}$. By the preceding remark

$$
u \leq \frac{M}{c_{0}}+\frac{M_{1}}{1-\beta_{0}} \text { on } R_{n} \times[0, T] .
$$

Similarly using

$$
w(x, t)=u(x, t)+\frac{M}{c_{0}}+\frac{M_{1}}{1-\beta_{0}}
$$


as a comparison function we deduce the inequality

$$
u \geq-\frac{M}{c_{0}}-\frac{M_{1}}{1-\beta_{0}} \text { on } R_{n} \times[0, T] .
$$

In the general case we use the transformation $u(x, t)=v(x, t) e^{-\left(c_{0} / 2\right) t}$.

4. In this section we derive an integral representation of the problem (1), (2) and (3) in an infinite strip and in a bounded cylinder.

TheOREM 9. Suppose that the assumptions $\left(\mathrm{C}_{1}\right)$ and $\left(\mathrm{C}_{2}\right)$ hold in $R_{n} \times$ $(0, T]$. Let $\beta_{i}(i=1, \cdots, N)$ and $\Psi$ be a continuous and bounded functions on $R_{n}$. Assume further that

$$
-1 \leq \sum_{i=1}^{N} \beta_{i}(x) \leq 0 \quad \text { and } \quad \beta_{i}(x) \leq 0(i=1, \cdots, N) \text { on } R_{n} .
$$

Then the unique solution in $C^{2,1}\left(R_{n} \times(0, T]\right) \cap C\left(R_{n}[0, T]\right) \cap E_{H}\left(R_{n} \times(0, T]\right)$ of the problem (1), (9) with $f \equiv 0$ is given by

$$
u(x, t)=\int_{R_{n}} P(x, t, y) \Psi(y) d y,
$$

for $(x, t) \in R_{n} \times(0, T]$, where $P(x, t, y)$ as a function of $(x, t)$ satisfies the equation $L P=0$ in $R_{n} \times(0, T]$ for almost all $y \in R_{n}$. Moreover $P$ satisfies the equation

$$
P(x, t, y)=-\int_{R_{n}} \Gamma(x, t ; z, 0) \sum_{i=1}^{N} \beta_{i}(z) P\left(z, T_{i}, y\right) d z+\Gamma(x, t ; y, 0)
$$

for all $(x, t) \in R_{n} \times(0, T]$ and almost all $y \in R_{n}$, where $\Gamma(x, t, y, 0)$ is the fundamental solution of $L u=0$.

Proof. Let $\Psi$ be a continuous and bounded function in $L^{2}\left(R_{n}\right)$. By Lemma 2 the unique solution of the problem (1), (9) in $C^{2,1}\left(R_{n} \times(0, T]\right)$ $\cap C\left(R_{n} \times[0, T]\right) \cap E_{H}\left(R_{n} \times(0, T]\right)$ is bounded on $R_{n} \times[0, T]$. We first prove that for each $\delta>0$ there exists a positive constant $C(\delta)$ such that

$$
|u(x, t)| \leq C(\delta)\left[\int_{R_{n}} \Psi(y)^{2} d y\right]^{1 / 2}
$$

on $R_{n} \times[\delta, T]$. To prove (12) we first assume that $-1<\beta_{0} \leq \sum_{i=1}^{N} \beta_{i}(x)$ $\leq 0$ on $R_{n}$, where $\beta_{0}$ is a positive constant. Consider the Cauchy problem for the homogeneous equation (1) with the initial condition

$$
z(x, 0)=-\sum_{i=1}^{N} \beta_{i}(x) u\left(x, T_{i}\right)+\Psi(x)
$$


on $R_{n}$. The unique solution $z$ in $E_{H}\left(R_{n} \times(0, T]\right)$ is given by

$$
z(x, t)=-\int_{R_{n}} \Gamma(x, t ; y, 0) \sum_{i=1}^{N} \beta_{i}(y) u\left(y, T_{i}\right) d y+\int_{R_{n}} \Gamma(x, t ; y, 0) \Psi(y) d y
$$

for all $(x, t) \in R_{n} \times(0, T]$ (Friedman [2], p. 26). Since $u$ is a solution of the same problem we obtain

$$
u(x, t)=-\int_{R_{n}} \Gamma(x, t ; y, 0) \sum_{i=1}^{N} \beta_{i}(y) u\left(y, T_{i}\right) d y+\int_{R_{n}} \Gamma(x, t ; y, 0) \Psi(y) d y
$$

for all $(x, t) \in R_{n} \times(0, T]$. Now it is well known that

$$
\int_{R_{n}} \Gamma(x, t ; y, 0) d y \leq 1
$$

for all $(x, t) \in R_{n} \times(0, T]$ and

$$
0<\Gamma(x, t ; y, 0) \leq C_{1} t^{-(n / 2)} e^{-\mathscr{e}(|x-y| 2) / t}
$$

for all $(x, t) \in R_{n} \times(0, T]$ and $y \in R_{n}$, where $C_{1}$ and $\mathscr{H}$ are positive constants (Friedman [2], p. 24). Applying the Hölder inequality we derive from (13), (14) and (15) that

$$
\max _{i=1, \cdots, N} \sup _{R_{n}}\left|u\left(x, T_{i}\right)\right| \leq \frac{C_{1}}{1-\beta_{0}} T_{k}^{-(n / 4)}\left[\int_{R_{n}} e^{-2 *|x|^{2}} d x\right]^{1 / 2}\left[\int_{R_{n}} \Psi(x)^{2} d x\right]^{1 / 2},
$$

where $T_{k}=\min _{i=1, \ldots, N} T_{i}$. Using again the representation (13) and the estimates (14), (15) and (16) we obtain

$$
|u(x, t)| \leq\left[\frac{\beta_{0}}{1-\beta_{0}} C_{1} C_{2}+C_{1} C_{3} t^{-(n / 4)}\right]\left[\int_{R_{n}} \Psi(x)^{2} d x\right]^{1 / 2}
$$

for all $(x, t) \in R_{n} \times(0, T]$, where

$$
C_{2}=T_{k}^{-(n / 4)}\left[\int_{R_{n}} e^{-2 \mathscr{F}|x|^{2}} d x\right]^{1 / 2} \quad \text { and } \quad C_{3}=\left[\int_{R_{n}} e^{-2 \mathscr{H}|x|^{2}} d x\right]^{1 / 2},
$$

and the estimate (12) easily follows. In the general case we use the transformation $u(x, t)=v(x, y) e^{-\left(c_{0} / 2\right) t}$. By (12) the mapping $\Psi \rightarrow u(x, t)$ defines a linear functional on $C_{b}\left(R_{n}\right) \cap L^{2}\left(R_{n}\right)$ continuous in $L^{2}$-norm. Here $C_{b}\left(R_{n}\right)$ denotes the space of continuous and bounded functions on $R_{n}$. Consequently the representation (10) follows from the Riesz representation theorem of a linear continuous functional on $L^{2}\left(R_{n}\right)$. To derive (11) observe that by (10) and (13) we have for every continuous bounded function $\Psi$ 


$$
\begin{aligned}
\int_{R_{n}} P(x, t, y) \Psi(y) d y=- & \int_{R_{n}} \Gamma(x, t ; y, 0) \sum_{i=1}^{N} \beta_{i}(y)\left[\int_{R_{n}} P\left(y, T_{i}, z\right) \Psi(z) d z\right] d y \\
& +\int_{R_{n}} \Gamma(x, t ; y, 0) \Psi(y) d y
\end{aligned}
$$

for $(x, t) \in R_{n} \times(0, T]$. Consequently if we fix $(x, t) \in R_{n} \times(0, T]$, applying Fubini's theorem, we obtain the identity (11) for almost all $y \in R_{n}$. Now choose $y \in R_{n}$ such that

$$
\int_{R_{n}} \Gamma(x, T ; z, 0) \sum_{j=1}^{N} \beta_{j}(z) P\left(z, T_{j}, y\right) d z
$$

is finite. Then by Theorem 1 in Watson [6] the integral

$$
\int_{R_{n}} \Gamma(x, t, z, 0) \sum_{j=1}^{N} \beta_{j}(z) P\left(z, T_{j}, y\right) d z
$$

is finite for all $(x, t) \in R_{n} \times(0, T]$ and represents a solution of the equation $L v=0$ in $R_{n} \times(0, T]$ and the last assertion of the theorem easily follows.

Similarly in the case of a bounded cylinder one can prove

TheOREm 10. Suppose the assumptions of Theorem 5 hold. Let $u$ be a solution of the problem (1), (2) and (3) with $\phi \equiv 0$ and $f \equiv 0$. Then

$$
u(x, t)=\int_{\Omega} p(x, t, y) \Psi(y) d y
$$

for all $(x, t) \in D$, where $p(x, t, y)$ as a function of $(x, t)$ satisfies the equation $L p=0$ for almost all $y \in \Omega$. Moreover

$$
p(x, t, y)=-\int_{\Omega} G(x, t ; z, 0) \sum_{i=1}^{N} \beta_{i}(z) p\left(z, T_{i}, y\right) d z+G(x, t ; y, 0)
$$

for $\operatorname{all}(x, t) \in D$ and almost all $y \in \Omega$, where $G(x, t ; y, 0)$ is the Green function for the operator $L$.

In the following theorem we shall show that $p$ and $P$ tend to infinity at the same rate as $t^{-(n / 2)}$.

TheOREm 11. Let the assumptions of Theorem 9 hold and let $D=\Omega$ $\times(0, T]$ be a bounded cylinder with $\partial \Omega \in C^{2+\alpha}$. Then there exists a positive constant $C$ such that

$$
p(x, t, y) \leq C \int_{\Omega} G(x, t ; z, 0) d z+G(x, t ; y, 0)
$$


for all $(x, t) \in D$ and almost all $y \in \Omega$, and moreover

$$
P(x, t, y) \leq C \int_{R_{n}} \Gamma(x, t ; z, 0) d z+\Gamma(x, t ; y, 0)
$$

for all $(x, t) \in R_{n} \times(0, T]$ and almost all $y \in R_{n}$, where $C$ depends on $C_{1}$ and $n$.

Proof. We first assume that $-1<\beta_{0} \leq \sum_{i=1}^{N} \beta_{i}(x) \leq 0$ on $\Omega$, where $\beta_{0}$ is a positive constant.

Let $\Psi$ be a continuous and non-negative function on $R_{n}$ with compact support in $\Omega$. It follows from Theorem 9,10 and the maximum principle that

$$
\int_{\Omega} p(x, t, y) \Psi(y) d y \leq \int_{R_{n}} P(x, t, y) \Psi(y) d y
$$

for all $(x, t) \in D$. Since $\Psi$ is an arbitrary non-negative function we deduce from the last inequality

$$
p(x, t, y) \leq P(x, t, y)
$$

for all $(x, t) \in D$ and almost all $y \in \Omega$. Fix $y$ in $\Omega$ such that the last inequality holds. Since $P\left(x, T_{i}, y\right)$ is continuous as a function of $x$ we get

$$
p\left(x, T_{i}, y\right) \leq \sup _{z \in \bar{\Omega}} P\left(z, T_{i}, y\right)<\infty \quad(i=1, \cdots, N)
$$

Using the identity (18), the estimate (15) and the obvious inequality $G(x, t ; y, 0) \leq \Gamma(x, t ; y, 0)$ for all $(x, t) \in R_{n} \times(0, T]$ and $y \in R_{n}$ we derive the estimate

$$
\max _{i=1, \cdots, N} \sup _{x \in \Omega} p\left(x, T_{i}, y\right) \leq \frac{C_{1} T_{k}^{-(n / 2)}}{1-\beta_{0}}, \quad \text { where } T_{k}=\min _{i=1, \cdots, N} T_{i} .
$$

Now applying again the identity (18) we obtain

$$
p(x, t, y) \leq \frac{C_{1} T_{k}^{-(n / 2)} \beta_{0}}{1-\beta_{0}} \int_{\Omega} G(x, t ; z, 0) d z+G(x, t ; y, 0)
$$

for all $(x, t) \in D$ and almost all $y \in \Omega$. In the general case we use the transformation $u(x, t)=v(x, t) e^{-\left(c_{0} / 2\right) t}$.

To prove (20) put $D_{m}(|x|<m) \times(0, T]$ and denote by $G_{m}(x, t ; y, 0)$ the Green function for the operator $L$. By the preceding result we have for every $m$ 


$$
p_{m}(x, t ; y) \leq C \int_{|z|<m} G(x, t ; z, 0) d z+G_{m}(x, t ; y, 0)
$$

for all $(x, t) \in D_{m}$ and almost all $y \in\{|x|<m\}$, where $p_{m}$ denotes " $p$-function" for the problem (1), (2) and (3) in $D_{m}$. By a standard argument one can prove that $\left\{G_{m}\right\}$ and $\left\{p_{m}\right\}$ are increasing sequences converging to $G$ and $p$ respectively and the result easily follows.

It follows from the proof of Theorem 9 (the inequality (12)) that the problem (1), (9) can be solved for $\Psi \in L^{2}\left(R_{n}\right)$, but this requires a new formulation of the condition (9).

We shall say that a function $u(x, t)$ defined on $R_{n} \times(0, T]$ has a parabolic limit at $x_{0}$ if there exists a number $b$ such that for all $\gamma>0$, we have

$$
\lim _{\substack{(x, t) \rightarrow\left(x_{0}, 0\right) \\\left|x-x_{0}\right|<r \sqrt{t}}} u(x, t)=b .
$$

We express this briefly by writing $p-\lim _{(x, t) \rightarrow\left(x_{0}, 0\right)} u(x, t)=b$ (see Chaborowski [1] p. 257).

Let $\Psi \in L^{2}\left(R_{n}\right)$. We shall say that a function $u$ belonging to $C^{2,1}\left(R_{n}\right.$ $\times(0, T])$ is a solution of the problem (1), (9) if it satisfies the equation (1) in $R_{n} \times(0, T]$ and

$$
p-\lim _{(x, t) \rightarrow(y, 0)} u(x, t)=-\sum_{i=1}^{N} \beta_{i}(y) u\left(y, T_{i}\right)+\Psi(y)
$$

for almost all $y \in R_{n}$.

Theorem 12. Suppose that the assumptions $\left(\mathrm{C}_{1}\right)$ and $\left(\mathrm{C}_{2}\right)$ hold in $R_{n} \times(0, T] . \quad$ Let $\beta_{i} \in C\left(R_{n}\right) \quad(i=1, \cdots, N)-1 \leq \sum_{i=1}^{N} \beta_{i}(x) \leq 0$ and $\beta_{i}(x)$ $\leq 0(i=1, \cdots, N)$ on $R_{n}$. Assume that $\Psi \in L^{2}\left(R_{n}\right)$ and that $f$ is a bounded function on $R_{n} \times[0, T]$ and Hölder continuous on every compact subset of $R_{n} \times[0, T]$. Then there exists a solution of the problem (1), (9).

Proof. Let $\left\{\Psi_{r}\right\}$ be a sequence of functions in $C\left(R_{n}\right)$ with compact supports which converges to $\Psi$ in $L^{2}\left(R_{n}\right)$. By Theorem 9 there exists a unique bounded solution $u_{r}$ in $C^{2,1}\left(R_{n} \times(0, T] \cap C\left(R_{n} \times[0, T]\right)\right.$ to the problem

$$
L u_{r}=f \text { in } R_{n} \times(0, T]
$$

and

$$
u_{r}\left(x,{ }^{r} 0\right)+\sum_{i=1}^{N} \beta_{i}(x) u_{r}\left(x, T_{i}\right)=\Psi_{r}(x) \quad \text { on } R_{n}
$$


It follows from (12) that

$$
\left|u_{r}(x, t)-u_{s}(x, t)\right| \leq C(\delta)\left\{\int_{R_{n}}\left[\Psi_{r}(x)-\Psi_{s}(x)\right]^{2} d x\right\}^{1 / 2}
$$

for all $(x, t) \in R_{n} \times[\delta, T]$. Hence $u_{r}(x, t)$ converges uniformly on $R_{n} \times[\delta, T]$ for every $\delta>0$ to a continuous function $u(x, t)$ on $R_{n} \times(0, T]$. As in the proof of Theorem 9 it is easy to establish the representation

$$
\begin{aligned}
u_{r}(x, t)= & -\int_{R_{n}} \Gamma(x, t ; y, 0) \sum_{i=1}^{N} \beta_{i}(y) u_{r}\left(y, T_{i}\right) d y \\
& +\int_{R_{n}} \Gamma(x, t ; y, 0) \Psi_{r}(y) d y-\int_{0}^{t} \int_{R_{n}} \Gamma(x, t ; y, \tau) f(y, \tau) d y d \tau
\end{aligned}
$$

for all $(x, t) \in R_{n} \times(0, T]$. Letting $r \rightarrow \infty$ we obtain

$$
\begin{aligned}
u(x, t)= & -\int_{R_{n}} \Gamma(x, t ; y, 0) \sum_{i=1}^{N} \beta_{i}(y) u\left(y, T_{i}\right) d y \\
& +\int_{R_{n}} \Gamma(x, t ; y, 0) \Psi(y) d y-\int_{0}^{t} \int_{R_{n}} \Gamma(x, t ; y, \tau) f(y, \tau) d y d \tau
\end{aligned}
$$

for $(x, t) \in R_{n} \times(0, T]$. Since $u\left(x, T_{i}\right)$ are bounded on $R_{n}$ it is easy to see that $u(x, t)$ satisfies the equation (1) in $R_{n} \times(0, T]$. It follows from Theorem 3.1 in Chabrowski [1] that

$$
p-\lim _{(x, t) \rightarrow(y, 0)} u(x, t)=-\sum_{i=1}^{N} \beta_{i}(y) u\left(y, T_{i}\right)+\Psi(y)
$$

for almost all $y \in R_{n}$.

5. In this section we briefly discuss the extensions of the previous results to the problem (1), (2) and $\left(3^{*}\right)$, where

$$
u(x, 0)+\sum_{i=1}^{\infty} \beta_{i}(x) u\left(x, T_{i}\right)=\Psi(x) \text { on } \Omega,
$$

with $T_{i} \in(0, T] i=1,2, \cdots$.

Throughout this section it is assumed that $\inf _{i} T_{i}>0$.

We being with the maximum principle.

LEMMA 3. Suppose that the assumption (A) holds in a bounded cylinder D. Let $c(x, t) \leq 0$ in $D$. Assume that $-1 \leq \sum_{i=1}^{\infty} \beta_{i}(x) \leq 0$ and $\beta_{i}(x) \leq 0$ $(i=1,2, \cdots)$ on $\Omega$. Let $u$ be a function in $C^{2,1}(D) \cap C(\bar{D})$ satisfying the following conditions

$$
L u \leq 0 \text { in } D
$$




$$
u(x, t) \geq 0 \quad \text { on } \Gamma
$$

and

$$
u(x, 0)+\sum_{i=1}^{\infty} \beta_{i}(x) u\left(x, T_{i}\right) \geq 0 \quad \text { on } \bar{\Omega},
$$

then $u \geq 0$ on $\bar{D}$.

Proof. Assume that $u<0$ at some point of $\bar{D}$. Then there exists a point $x_{0} \in \Omega$ such that $u\left(x_{0}, 0\right)=\min _{\bar{\Omega}} u(x, t)<0$. Consequently

$$
u\left(x_{0}, 0\right)\left(1+\sum_{i=1}^{\infty} \beta_{i}\left(x_{0}\right)\right) \geq 0 \text {. }
$$

Hence $u\left(x_{0}, 0\right) \geq 0$ provided $\sum_{i=1}^{\infty} \beta_{i}\left(x_{0}\right)+1>0$ and we get a contradiction.

It remains to consider the case $\sum_{i=1}^{\infty} \beta_{i}\left(x_{0}\right)=-1$. Let $T_{0}=\inf _{i} T_{i}$. There exists $S \in\left[T_{0}, T\right]$ such that $u\left(x_{v}, S\right)=\min _{T_{0 \leq t \leq T}} u\left(x_{0}, t\right)$. Hence

$$
u\left(x_{0}, 0\right) \geq-\sum_{i=1}^{\infty} \beta_{i}\left(x_{0}\right) u\left(x_{0}, T_{i}\right) \geq-u\left(x_{0}, S\right) \sum_{i=1}^{\infty} \beta_{i}\left(x_{0}\right)=u\left(x_{0}, S\right)
$$

and we get a contradiction.

TheOREm 13. Suppose that the assumption (A) holds in a bounded cylinder. Let $c(x, t) \leq 0$ on $D$ and $\sum_{i=1}^{\infty}\left|\beta_{i}(x)\right| \leq 1$ on $\Omega$. Then the problem (1), (2) and (3*) has at most one solution in $C^{2,1}(D) \cap C(\bar{D})$.

Proof. Let $u$ be a solution of the homogeneous problem

$$
\begin{aligned}
L u=0 & \text { in } D, \\
u(x, t)=0 & \text { on } \Gamma
\end{aligned}
$$

and

$$
u(x, 0)+\sum_{i=1}^{\infty} \beta_{i}(x) u\left(x, T_{i}\right)=0 \quad \text { on } \Omega .
$$

Suppose that $u \neq 0$. As in the proof of Theorem 4 we may assume that there exists a point $x_{v} \in \Omega$ such that

$$
u\left(x_{0}, 0\right)=\min _{\bar{D}} u(x, t)<0 . \quad \text { Let }\left|u\left(x_{0}, \kappa\right)\right|=\max _{T_{0} \leq t \leq T}\left|u\left(x_{0}, T\right)\right|,
$$

where $T_{0}=\inf _{i} T_{i}$ and $\kappa \in\left[T_{0}, T\right]$. Then

$$
\left|u\left(x_{0}, 0\right)\right| \leq\left|u\left(x_{0}, \kappa\right)\right| \sum_{i=1}^{\infty}\left|\beta_{i}\left(x_{0}\right)\right| \leq\left|u\left(x_{0}, \kappa\right)\right| .
$$


We must assume that $u\left(x_{0}, k\right)>0$. Hence there exists a point $x_{1} \in \Omega$ such that $u\left(x_{1}, 0\right)=\max _{\bar{D}} u(x, t)>0$. Let $\left|u\left(x_{1}, S\right)\right|=\max _{T_{0} \leq t \leq T}\left|u\left(x_{1}, S\right)\right| . \quad$ It is obvious that

$$
u\left(x_{1}, 0\right) \leq\left|u\left(x_{1}, S\right)\right| .
$$

Now considering two cases $u\left(x_{1}, 0\right) \leq\left|u\left(x_{0}, 0\right)\right|$ and $\left|u\left(x_{0}, 0\right)\right|<u\left(x_{1}, 0\right)$ we arrive at a contradiction (for details see the proof of Theorem 3 ).

We shall now state analogues of Theorems 5 and 8 .

THEOREm 14. Suppose that the assumptions $\left(\mathrm{A}_{1}\right)$ and $\left(\mathrm{A}_{2}\right)$ hold in a bounded cylinder $D$ with $\partial \Omega \in C^{2+\alpha}$. Let $c(x, t) \leq-c_{0}$ in $D$, where $c_{0}$ is a positive constant and assume that $\beta_{i} \in C(\bar{\Omega})(i=1,2, \cdots), \beta_{i}(x) \leq 0(i=1$, $2, \cdots)$ and $-1 \leq \sum_{i=1}^{\infty} \beta_{i}(x) \leq 0$ on $\Omega$ and that the series $\sum_{i=1}^{\infty} \beta_{i}(x)$ is uniformly convergent on $\bar{\Omega}$. Assume finally that $f$ is a Hölder continuous function on $D, \phi$ and $\Psi$ are continuous function on $\Gamma$ and $\bar{\Omega}$ respectively and moreover

$$
\phi(x, 0)+\sum_{i=1}^{\infty} \beta_{i}(x) \phi\left(x, T_{i}\right)=\Psi(x) \quad \text { on } \partial \Omega .
$$

Then there exists a unique solution in $C^{2,1}(D) \cap C(\bar{D})$ of the problem (1), (2) and (3*).

TheORem 15. Let the assumptions $\left(\mathrm{C}_{1}\right)$ and $\left(\mathrm{C}_{2}\right)$ hold. Assume that $\beta_{i} \in C\left(R_{n}\right)(i=1,2, \cdots), \beta_{i}(x) \leq 0(i=1,2, \cdots)$ and $-1 \leq \sum_{i=1}^{\infty} \beta_{i}(x) \leq 0$ on $R_{n}$ and that the series $\sum_{i=1}^{\infty} \beta_{i}(x)$ is uniformly convergent on $R_{n}$. If $f$ is $a$ bounded on $R_{n} \times[0, T]$ and Hölder continuous function on every compact subset of $R_{n} \times[0, T]$ and $\Psi$ is a continuous and bounded function on $R_{n}$, then there exists a unique solution in $E_{H}\left(R_{n} \times(0, T]\right) \cap C^{2,1}\left(R_{n} \times(0, T]\right) \cap$ $C\left(R_{n} \times[0, T]\right)$ of the equation (1) satisfying the condition

$$
u(x, 0)+\sum_{i=1}^{\infty} \beta_{i}(x) u\left(x, T_{i}\right)=\Psi(x) \text { on } R_{n} .
$$

The proof of Theorem 14 and 15 are similar to those of Theorems 5 and 8.

One can easily prove that under the assumptions of Theorems 15 , the solution in $E_{H}\left(R_{n} \times(0, T]\right)$ of the problem (1), (9*) is bounded on $R_{n} \times$ $[0, T]$.

Remark. If 0 is an accumulation point of the sequence $\left\{T_{i}\right\}$ then the Lemma 3 remains true provided $\sum_{i=1}^{\infty} \beta_{i}(x)+1>0$ and $\beta_{i}(x) \leq 0(i=1$, $2, \cdots)$ on $R_{n}$. 


\section{REFERENCES}

[1] J. Chabrowski, Representation theorems for parabolic systems, J. Austral. Math. Soc. Ser. A, 32 (1982), 246-288.

[2] A. Friedman, Partial differential equations of parabolic type, Prentice-Hall, Englewood Cliffs, N. J. 1964.

[ 3 ] A. A. Kerefov, Non-local boundary value problems for parabolic equation, Differentsial'nye Uravnenija, 15 (1979), 52-55.

[4] M. Krzyzański, Sur les solutions de l'équation linéaire du type parabolique déterminées par les conditions initiales, Ann.Soc. Polon. Math., 18 (1945), 145-156, and note complémentaire, ibid., 10 (1947), 7-9.

[ 5 ] M. H. Protter, H. F. Weinberger, Maximum principles in differential equations, Prentice-Hall, Englewood Cliffs, N. J. 1967.

[6 ] P. N. Vabishchevich, Non-local parabolic problems and the inverse heat-conduction problem, Differentsial'nye Uravnenija, 17 (1981), 761-765.

[ 7 ] N. A. Watson, Uniqueness and representation theorems for parabolic equations, J. London Math. Soc. (2), 8 (1974), 311-321.

University of Queensland, Department of Mathematics,

St. Lucia Queensland 406\%,

Australia. 